\title{
Reversed exchange-bias effect associated with magnetization reversal in the weak ferrimagnet $\mathrm{LuFe}_{0.5} \mathrm{Cr}_{0.5} \mathrm{O}_{3}$
}

\author{
I. Fita, ${ }^{1,}$ V. Markovich, ${ }^{2}$ A. S. Moskvin, ${ }^{3}$ A. Wisniewski, ${ }^{1}$ R. Puzniak, ${ }^{1}$ P. Iwanowski, ${ }^{1}$ C. Martin, ${ }^{4}$ A. Maignan, ${ }^{4}$ \\ Raúl E. Carbonio, ${ }^{5}$ M. U. Gutowska, ${ }^{1}$ A. Szewczyk, ${ }^{1}$ and G. Gorodetsky ${ }^{2}$ \\ ${ }^{1}$ Institute of Physics, Polish Academy of Sciences, Aleja Lotnikow 32/46, PL-02668 Warsaw, Poland \\ ${ }^{2}$ Department of Physics, Ben-Gurion University of the Negev, P.O. Box 653, 84105 Beer-Sheva, Israel \\ ${ }^{3}$ Ural Federal University, Ekaterinburg, Russia \\ ${ }^{4}$ Laboratoire CRISMAT, UMR 6508, ENSICAEN, 14050 Caen Cedex, France \\ ${ }^{5}$ INFIQC (CONICET-Universidad Nacional de Córdoba), Departamento de Fisicoquímica, Facultad de Ciencias Químicas, \\ Universidad Nacional de Córdoba, Haya de la Torre esq. Medina Allende, Ciudad Universitaria, X5000HUA Córdoba, Argentina
}

(Received 9 October 2017; revised manuscript received 4 March 2018; published 23 March 2018)

\begin{abstract}
The exchange-bias (EB) effect with sign reversal was found in $\mathrm{LuFe}_{0.5} \mathrm{Cr}_{0.5} \mathrm{O}_{3}$ ferrite-chromite, which is a weak ferrimagnet below $T_{\mathrm{N}}=265 \mathrm{~K}$, exhibiting antiparallel orientation of the ferromagnetic (FM) moments of the Fe and $\mathrm{Cr}$ sublattices due to opposite sign of the Fe-Cr Dzyaloshinskii vector, as compared to that of the Fe-Fe and $\mathrm{Cr}$-Cr. The weak FM moments of the studied compound compensate each other at temperature $T_{\text {comp }}=230 \mathrm{~K}$, leading to the net magnetic moment reversal and to observed negative magnetization, at moderate applied fields, below $T_{\text {comp. }}$. Both vertical and horizontal shifts from the origin were gotten in the field-cooled magnetization hysteresis loops. The EB sign was found to be positive below $T_{\text {comp }}$ and negative above $T_{\text {comp }}$, with nonmonotonic dependence on cooling field $H_{\text {cool }}$. It sharply increases at small values of magnetic fields up to $H_{\text {cool }} \sim 1 \mathrm{kOe}$, then remains almost unchanged in the range 1-30 kOe and strongly decreases with further increase of $H_{\text {cool }}$. This unusual behavior results from the competition of various Dzyaloshinskii-Moriya interactions between $\mathrm{Fe}^{3+}$ and $\mathrm{Cr}^{3+}$ ions.
\end{abstract}

DOI: 10.1103/PhysRevB.97.104416

\section{INTRODUCTION}

The exchange-bias (EB) effect discovered by Meiklejohn and Bean [1] is at present a key instrument for practical applications in magnetic memory and spintronics. The EB, found in a variety of magnetic heterostructures, is associated mainly with an interfacial exchange interaction between strongly anisotropic antiferromagnetic (AFM) and soft ferromagnetic (FM) phases, leading to a shift of magnetization hysteresis loop from the origin [2,3]. Recently, EB has been observed in some ferrimagnets (FIMs) that exhibit spontaneous magnetization reversal or negative magnetization, occurring when the net magnetic moment becomes oriented opposite to the orientation of the applied field [4-12]. An especially interesting finding is that EB of compensated FIMs, composed of two AFM coupled sublattices with opposite weak FM moments, reverses its sign at crossing the compensation temperature $T_{\text {comp }}$. Moreover, EB can be modified by applied magnetic field and change in temperature [6-9]. This striking feature is valuable for device applications, such as thermally assisted magnetic random access memories [13] and, on the other hand, it may help to understand the microscopic origin of the EB anisotropy. In this context, the observation of EB in single-phase and single-crystal FIM materials [4-11] suggests the very different origin of EB from that of the traditional EB involving the FM/AFM interface. Such type of EB, which originates from

\footnotetext{
*Corresponding author: ifita@ifpan.edu.pl
}

the intrinsic exchange coupling within the unit cell, should be referred as the atomic EB [14].

In this paper, we present the EB effect in a solid solution of two weak ferromagnetic materials, namely orthoferriteorthochromite $\mathrm{LuFe}_{0.5} \mathrm{Cr}_{0.5} \mathrm{O}_{3}$, which is a representative of exceptional class of FIM perovskite oxides with two magnetic transition metal ions randomly distributed at the B sites and one nonmagnetic cation at the A sites.

The unconventional concentration and temperature dependence of magnetization with compensation effects in singlecrystalline mixed orthoferrite-orthochromites was observed and explained by Kadomtseva et al. forty years ago [15]. The unusual behavior of these compounds was explained within the molecular field approximation (MFA) [15-18] using a simple model considering the isotropic superexchange $V_{\mathrm{ex}}=$ $\sum_{m>n} J_{m n}\left(\vec{S}_{m} \cdot \vec{S}_{n}\right)$ and the antisymmetric DzyaloshinskiiMoriya $(\mathrm{DM}) V_{\mathrm{DM}}=\sum_{m>n}\left(\vec{d}_{m n} \cdot\left[\vec{S}_{m} \times \vec{S}_{n}\right]\right)$ interactions, where $J_{m n}$ are the exchange integrals and $\vec{d}_{m n}$ are the Dzyaloshinskii vectors. Experimental values of the exchange integrals $J_{\mathrm{FeFe}}$ and $J_{\mathrm{CrCr}}$ were estimated from the Néel temperatures of $R \mathrm{FeO}_{3}$ and $R \mathrm{CrO}_{3}$ ( $R$ : rare earth), while $J_{\mathrm{FeCr}}$ was determined from fitting of the temperature dependence of the hyperfine local field on ${ }^{57} \mathrm{Fe}$ nuclei in $\mathrm{YFe}_{0.01} \mathrm{Cr}_{0.99} \mathrm{O}_{3}$ [19]. All the integrals are positive in sign, i.e., referring to antiferromagnetic interactions, for instance in $\mathrm{YFe}_{1-x} \mathrm{Cr}_{x} \mathrm{O}_{3}$ : $J_{\mathrm{FeFe}} / k_{\mathrm{B}}=18.3 \mathrm{~K}, J_{\mathrm{CrCr}} / k_{\mathrm{B}}=9.4 \mathrm{~K}, J_{\mathrm{FeCr}} / k_{\mathrm{B}}=6.7 \mathrm{~K}$ (see also Ref. [20]). According to theoretical predictions [21,22], Dzyaloshinskii vectors $\vec{d}_{\mathrm{FeFe}}$ and $\vec{d}_{\mathrm{CrCr}}$ possess the same sign; however, it is opposite to the sign of the $\vec{d}_{\mathrm{FeCr}}$ vector: 

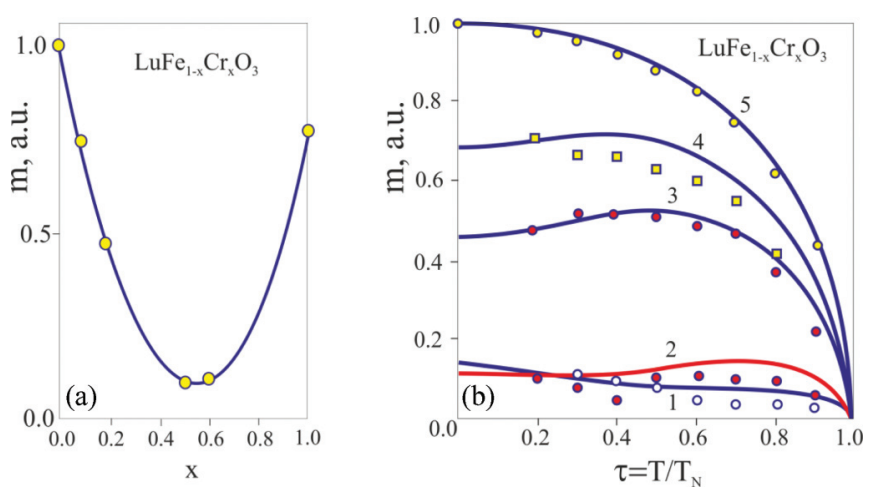

FIG. 1. (a) Concentration dependence of the low-temperature magnetization/magnetic moment (recorded at $T=77 \mathrm{~K}$ ) in $\mathrm{LuFe}_{1-x} \mathrm{Cr}_{x} \mathrm{O}_{3}$ : Experimental data (circles) and MFA calculations (solid curve). (b) Temperature dependence of magnetization/magnetic moment in $\mathrm{LuFe}_{1-x} \mathrm{Cr}_{x} \mathrm{O}_{3}$ : Experimental data (circles) are given for $x=0.6(1), 0.5$ (2), 0.2 (3), 0.1 (4), 0.0 (5); solid curves are the MFA calculations. Both figures are reprinted from Ref. [16].

$\vec{d}_{\mathrm{FeFe}} \uparrow \uparrow \vec{d}_{\mathrm{CrCr}} \uparrow \downarrow \vec{d}_{\mathrm{FeCr}}$. This leads to unconventional character of the concentration and temperature dependence of magnetization $M(x, T)$. Low-temperature magnetization sharply falls with substitution of $\mathrm{Fe}$ by $\mathrm{Cr}$ in $\mathrm{YFeO}_{3}$ or $\mathrm{Cr}$ by $\mathrm{Fe}$ in $\mathrm{YCrO}_{3}$ down to very small values near $x=0.5$.

Experimental studies of the single-crystalline samples of the mixed orthoferrites-orthochromites $\mathrm{YFe}_{1-x} \mathrm{Cr}_{x} \mathrm{O}_{3}$ for a wide range of substitution performed by Kadomtseva et al. [15] did confirm theoretical predictions regarding the signs of the Dzyaloshinskii vectors and revealed the weak ferrimagnetic behavior due to a competition of $\mathrm{Fe}-\mathrm{Fe}, \mathrm{Cr}-\mathrm{Cr}$, and $\mathrm{Fe}-\mathrm{Cr}$ DM coupling with antiparallel orientation of the mean weak ferromagnetic moments of $\mathrm{Fe}$ and $\mathrm{Cr}$ subsystems. In contrast to $\mathrm{YFeO}_{3}$ and $\mathrm{YCrO}_{3}$, which are weak ferromagnets with the main $\mathrm{G}_{x} \mathrm{~F}_{z}$-type magnetic structure below $T_{\mathrm{N}}$, the orthoferritesorthochromites $\mathrm{YFe}_{1-x} \mathrm{Cr}_{x} \mathrm{O}_{3}$, which should be referred as weak ferrimagnets [15], reveal full or partial $\mathrm{G}_{x} \mathrm{~F}_{z}-\mathrm{G}_{z} \mathrm{~F}_{x}$ type spin reorientation for a wide range of substitution. This unexpected behavior, which is typical for orthoferrites with magnetic rare-earth ions $(\mathrm{Er}, \mathrm{Tm}, \mathrm{Dy}, \ldots)$, was attributed mainly to a strong decrease of the DM contribution to magnetic anisotropy in the $a c$ plane for $x=0.5-0.6$ [18].

In contrast to the behavior of the yttrium system, the lutetium orthoferrite-orthocromites $\mathrm{LuFe}_{1-x} \mathrm{Cr}_{x} \mathrm{O}_{3}(x=0.0$, $0.1,0.2,0.5,0.6$, and 1.0) reveal the main $\mathrm{G}_{x} \mathrm{~F}_{z}$-type magnetic structure without signatures of the spontaneous spinreorientation transition [16]. Concentration dependence of the low-temperature (77 K) ZFC magnetization and temperature dependence of magnetization are shown in Fig. 1. Both concentration and temperature dependencies of magnetization for $\mathrm{LuFe}_{1-x} \mathrm{Cr}_{x} \mathrm{O}_{3}$ are well described within a simple MFA model with constant value of the parameter $\delta=\frac{d_{\mathrm{CrFe}}}{J_{\mathrm{CF}}}: \frac{d_{\mathrm{FeFe}}}{J_{\mathrm{FF}}}=-1.5$. It is worth noting that the previous measurements [16] did not reveal compensation points; however, one cannot exclude the existence of the compensation point for the doping level around $x=0.5$.

Revival of the interest in orthoferrites-orthochromites, first of all in $\mathrm{YFe}_{1-x} \mathrm{Cr}_{x} \mathrm{O}_{3}$, in the last decade [20,23-26] was stimulated by perspectives to find the magnetization reversal and $\mathrm{EB}$ effects, that are very promising in view of possible applications. Both effects have been recently reported for several ferrite-chromite systems such as $\mathrm{YFe}_{0.5} \mathrm{Cr}_{0.5} \mathrm{O}_{3}$ [27,28], $\mathrm{Y}_{1-x} \mathrm{Ho}_{x} \mathrm{Fe}_{0.5} \mathrm{Cr}_{0.5} \mathrm{O}_{3}$ ( $\left.x=0,0.05,0.1\right)$ [29], $\mathrm{LaCr}_{1-x} \mathrm{Fe}_{x} \mathrm{O}_{3}$ $\left(x=0.4\right.$ to 0.6) [30], $\mathrm{NdCr}_{1-x} \mathrm{Fe}_{x} \mathrm{O}_{3}(x=0.05-0.2)$ [31], and $\mathrm{NdFe}_{0.5} \mathrm{Cr}_{0.5} \mathrm{O}_{3}$ [32], and were discussed in terms of competition between the DM interaction and single-ion magnetic anisotropy [27-31]. It should be noted, however, that in these publications hysteresis loops were recorded with very small values of maximal field $H_{\max }$ of 5-10 kOe [27,28,30,31] and these curves do not show any reversible part, where the ascending and descending branches of the loops coincide, so they actually represent minor hysteresis loops. It is well known that such curves are naturally displaced from the origin which, however, is irrelevant to the exchange-bias phenomenon $[33,34]$. Only in Refs. [29,32] hysteresis loops were recorded at higher maximum field values, $H_{\max }= \pm 30 \mathrm{kOe}$ [29] and $\pm 70 \mathrm{kOe}$ [32], but the presented results cause also deep doubt in the presence of the true EB effect, since hysteresis loops remain unsaturated [33-35]. Hysteresis loops may properly reveal the presence of the EB effect only when they are saturated or effectively saturated. Geshev [33,34] proposed that the presence of "true" EB in mostly AFM systems in the absence of the saturation may be properly verified by recordings of the "effectively saturated" hysteresis loops. A system is effectively saturated when the ascending and descending branches of the hysteresis loop coincide at fields higher than the anisotropy field [33,34]. Recently, Harres et al. [35] suggested a few independent criteria for discrimination between nonsaturated (minor) and saturated (major) or effectively saturated hysteresis loops.

Most recently, Pomiro et al. [26] observed the spontaneous magnetization reversal in polycrystalline $\mathrm{LuFe}_{0.5} \mathrm{Cr}_{0.5} \mathrm{O}_{3}$ below $T_{\mathrm{N}}=290 \mathrm{~K}$ at $T_{\text {comp }}=224 \mathrm{~K}$. However, in the composition range near $x=0.5$ the magnetization reversal is unstable. Indeed, classical Monte Carlo simulation for $\mathrm{LuFe}_{1-x} \mathrm{Cr}_{x} \mathrm{O}_{3}$ [20] does not support experimental finding of the magnetization reversal in $\mathrm{LuFe}_{0.5} \mathrm{Cr}_{0.5} \mathrm{O}_{3}$. However, the magnetization curve calculated for $x=0.4$ reproduces well the experimental curve reported for $\mathrm{LuFe}_{0.5} \mathrm{Cr}_{0.5} \mathrm{O}_{3}$. Below the Néel temperature the magnetization due to the $\mathrm{Fe}^{3+}$ ions aligns in the direction of the magnetic field, while the magnetization due to the $\mathrm{Cr}^{3+}$ ions is opposite to the external field. The different temperature dependencies of the magnetization of $\mathrm{Fe}^{3+}$ and $\mathrm{Cr}^{3+}$ ions turn into the magnetization reversal; in particular at low temperatures the contribution to the magnetization of the $\mathrm{Cr}^{3+}$ ions becomes larger than the contribution of the $\mathrm{Fe}^{3+}$ ions. Hereafter, we present results of comprehensive magnetic measurements that allowed us to evidence and elucidate the magnetization reversal and EB effect in $\mathrm{LuFe}_{0.5} \mathrm{Cr}_{0.5} \mathrm{O}_{3}$. The strong positive $\mathrm{EB}$ effect observed below $T_{\text {comp }}=240 \mathrm{~K}$ down to $10 \mathrm{~K}$ results from the net weak FM moment reversal at modest positive applied field, yielding hence the negative remanence in the course of the magnetization loop.

\section{EXPERIMENTAL DETAILS}

A polycrystalline $\mathrm{LuFe}_{0.5} \mathrm{Cr}_{0.5} \mathrm{O}_{3}$ sample was prepared by the wet chemical method described thoroughly in Ref. [26]. The studied sample was single phase with the expected Pnma 


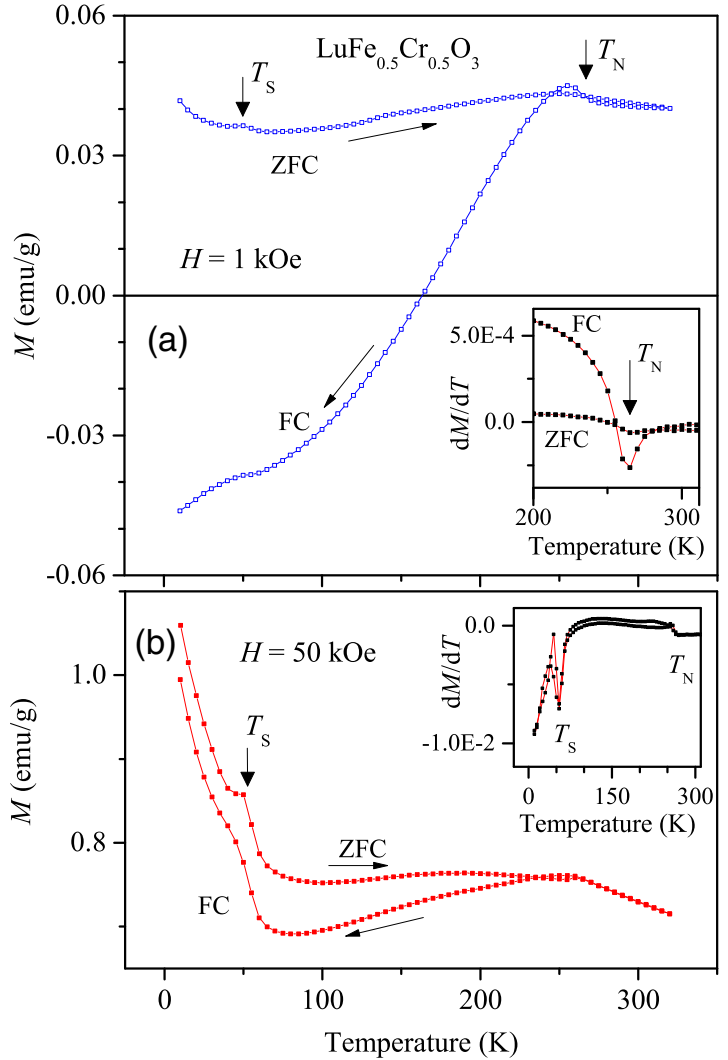

FIG. 2. Temperature dependence of magnetization of $\mathrm{LuFe}_{0.5} \mathrm{Cr}_{0.5} \mathrm{O}_{3}$ measured at (a) $H=1 \mathrm{kOe}$ and (b) $H=50 \mathrm{kOe}$ for ZFC and FC modes. The insets show derivative $d M / d T$ vs $T$.

orthorhombic structure with lattice parameters $a=5.1967 \AA$, $b=5.5270 \AA$, and $c=7.5195 \AA$, determined using x-ray diffraction (XRD), and with the $\mathrm{Fe} / \mathrm{Cr}$ occupancy at the $4 b$ sites of $0.47(2) / 0.53(2)$, obtained from neutron powder diffraction (NPD) [26]. Detailed temperature evolution of the crystal and magnetic structures in the range 4-300 K, as well as basic magnetization characteristics of the $\mathrm{LuFe}_{0.5} \mathrm{Cr}_{0.5} \mathrm{O}_{3}$ sample, were already published [26]. For the present magnetic studies, the $\mathrm{LuFe}_{0.5} \mathrm{Cr}_{0.5} \mathrm{O}_{3}$ powder, formerly studied by XRD and NPD, was compacted into pellets under a pressure of $5 \mathrm{kbar}$. The magnetization measurements for the temperature range $10-320 \mathrm{~K}$ in magnetic field up to $90 \mathrm{kOe}$, as well as supplementary specific heat studies for the temperature range 2-300 K, in zero magnetic field and in the field of $90 \mathrm{kOe}$ were performed by using the vibrating sample magnetometer and specific heat options of the physical property measurement system (PPMS-9T) made by Quantum Design.

\section{RESULTS AND DISCUSSION}

Figure 2 presents the temperature dependence of magnetization of $\mathrm{LuFe}_{0.5} \mathrm{Cr}_{0.5} \mathrm{O}_{3}$ measured at (a) $\mathrm{H}=1 \mathrm{kOe}$ and (b) $H=50 \mathrm{kOe}$ for zero-field-cooling (ZFC) and field-cooling (FC) modes. Figure 3 shows the remanent magnetization $M_{\mathrm{r}}$ vs $T$ recorded on warming at $H=0$, after FC in cooling field $H_{\text {cool }}=5,+50$, and $-50 \mathrm{kOe}$. The $M(T)$ and $M_{\mathrm{r}}(T)$ curves show an abnormal feature that may be attributed to

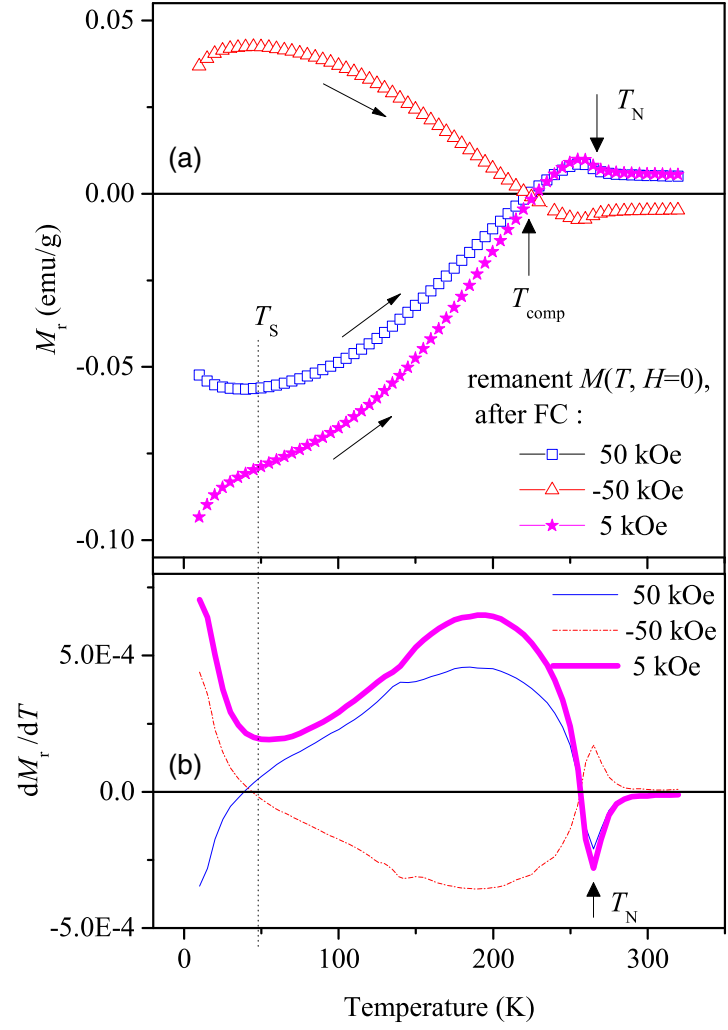

FIG. 3. (a) Remanent magnetization $M_{\mathrm{r}}$ vs $T$ recorded on warming at $H=0$, after $\mathrm{FC}$ in $H_{\text {cool }}=5,+50$, and $-50 \mathrm{kOe}$, and (b) corresponding derivatives $d M_{\mathrm{r}} / d T$ vs $T$.

the reversal of the weak FM moment, which dominates the magnetic behavior of $\mathrm{LuFe}_{0.5} \mathrm{Cr}_{0.5} \mathrm{O}_{3}$.

The magnetic system of the sample, frozen in its demagnetized state following the ZFC mode, represents an ensemble of the nearly orthogonal to each other AFM vectors and weak FM moments pointed in random directions. Hence, the ZFC magnetization is positive (see Fig. 2), exhibiting mainly the contribution from the AFM component while the FM moments are compensated. In a wide low-temperature range, the ZFC magnetization is larger than the $\mathrm{FC}$ one, even at the field of $50 \mathrm{kOe}$. The low-temperature FC magnetization is negative at $1 \mathrm{kOe}$, which indicates that its direction is opposite to the applied field. The phenomenon of spontaneous FM moment reversal is well demonstrated in Fig. 3 by the mirror-like behavior of the two $M_{\mathrm{r}}(T)$ curves, recorded at $H=0$ after cooling down to $10 \mathrm{~K}$ in two opposing fields, +50 and $-50 \mathrm{kOe}$. In both cases, the $M_{\mathrm{r}}$ is aligned opposite to the direction of $H_{\text {cool }}$ below temperature $T_{\text {comp }}=230 \mathrm{~K}$, at which the FM moments of Fe and $\mathrm{Cr}$ sublattices compensate each other. Due to their different temperature dependencies, the FM Fe moment prevails over the $\mathrm{Cr}$ one above $T_{\text {comp }}$, while the FM of $\mathrm{Cr}$ becomes larger than that of Fe below $T_{\text {comp }}$ [20]. The weak FM moment emerges consequently for the canted AFM spin ordering at the Néel temperature $T_{\mathrm{N}}=265 \mathrm{~K}$, defined by the minimum in derivative $d M / d T$ [inset of Figs. 2(a) and 3(b)]. Nevertheless, the $M_{\mathrm{r}}$ remains nonzero far above $T_{\mathrm{N}}$, in agreement with the NPD data suggesting that short-range ordered regions, whose magnetism is dominated by the $\mathrm{Fe}^{3+}-\mathrm{O}-\mathrm{Fe}^{3+}$ superexchange, exist above $T_{\mathrm{N}}$ [26]. A good agreement is observed in the 
values of $T_{\mathrm{N}}$, equal to $265 \mathrm{~K}$ as determined in this work, to be compared with $260 \mathrm{~K}$ in Ref. [26], corresponding to the maximum of the FC magnetic susceptibility, though the magnetic order started at $T_{\mathrm{N} 1} \approx 290 \mathrm{~K}$. The anomalies in $M(T)$ around $T_{\mathrm{S}}=50 \mathrm{~K}$ (Fig. 2) are particularly well pronounced by a sharp rise in magnetization with lowering temperature, accompanied by peculiar features well seen in $M_{\mathrm{r}}$ and especially in $d M_{\mathrm{r}} / d T$ at $H=50 \mathrm{kOe}$ (see Fig. 3). They most likely point to the field-induced spin-reorientation transitions in the studied weak FIM sample which represent an interplay of spin-flop transitions such as $\mathrm{G}_{z}-\mathrm{G}_{z x}$ in crystallites with the AFM vector oriented predominantly collinearly with external field and first-order transitions in crystallites with the FM vector oriented predominantly opposite to external field when "... the sublattice magnetization vectors flip over in such a way that the vector product $M_{1} \times M_{2}$ changes sign" [36] with an "overturning" of the weak FM and lining up along external field. Critical fields for these FM overturning transitions depend strongly on both local Dzyaloshinskii $\left(H_{\mathrm{D}}\right)$ and anisotropy $\left(H_{\mathrm{A}}\right)$ fields and could possibly be a power function of the $H_{\mathrm{A}} / H_{\mathrm{D}}$ ratio [36]. In rather strong magnetic field, both transitions would lead to a positive contribution, parallel to the field, to the overall FM and to a decrease in $M_{\mathrm{r}}$, while for small or moderate fields we arrive at a competition between the AFM spin-flop susceptibility and Zeeman contributions. This effect is clearly demonstrated in Fig. 3(a), where the $M_{\mathrm{r}}$ is found to decrease or increase below $T_{\mathrm{S}}$ depending on the value of cooling field. However, strictly stated, Figs. 2 and 3 demonstrate the temperature-driven spinreorientation transition under constant external magnetic field.

Figure 4(a) shows the magnetization hysteresis loops of $\mathrm{LuFe}_{0.5} \mathrm{Cr}_{0.5} \mathrm{O}_{3}$ at $10 \mathrm{~K}$ measured between $\pm 50 \mathrm{kOe}$ in $\mathrm{ZFC}$ mode and with cooling fields of $+5 \mathrm{kOe}$ and $-5 \mathrm{kOe}$. The loops demonstrate an important feature: while the ZFC loop is entirely symmetric, the FC ones exhibit a substantial EB effect, namely, both vertical and horizontal shifts from the origin. In accordance with Ref. [37], the EB characteristics are defined as the remanence asymmetry $M_{\mathrm{EB}}=\left(M_{1}+M_{2}\right) / 2$ and EB field $H_{\mathrm{EB}}=\left(H_{1}+H_{2}\right) / 2$, where $M_{1}$ and $M_{2}$ are the remnant magnetizations at the field decreasing and increasing branches of the loop, respectively, and $H_{1}$ and $H_{2}$ are the respective coercive fields [inset in Fig. 4(a)]. It appears that the positive $H_{\text {cool }}$ shifts the loop down causing the positive exchange-bias field $H_{\mathrm{EB}}$, while an applied negative $H_{\text {cool }}$ induces a negative $H_{\mathrm{EB}}$. It is worth noting that $M_{\mathrm{EB}}$ is close to $M_{\mathrm{r}}(10 \mathrm{~K})$, since the loop is relatively narrow [see Fig. 3(a)]. The loops demonstrate the positive $\mathrm{EB}$ which is known to happen in conventional AFM-FM interface systems, such as $\mathrm{FeF}_{2}-\mathrm{Fe}$ bilayers, in the case of a specific spin configuration arising, when $H_{\text {cool }}$ is large enough to overcome the AFM interfacial coupling [38]. Interestingly, these systems exhibit also a downwards vertical shift of the hysteresis loop, similar to that seen in Fig. 4(a), which indicates that the induced moment direction is opposite to the direction of the cooling field [39]. However, the downwards magnetization shift in $\mathrm{FeF}_{2}-\mathrm{Fe}$ bilayers is due to the pinned interfacial spins that are not rotated by the applied field and it always correlates with the negative EB at small cooling fields [39], in contrast to the effect observed for the $\mathrm{LuFe}_{0.5} \mathrm{Cr}_{0.5} \mathrm{O}_{3}$ ferrimagnet. It was also found that the sign of EB is determined by the direction of the domain magnetization,

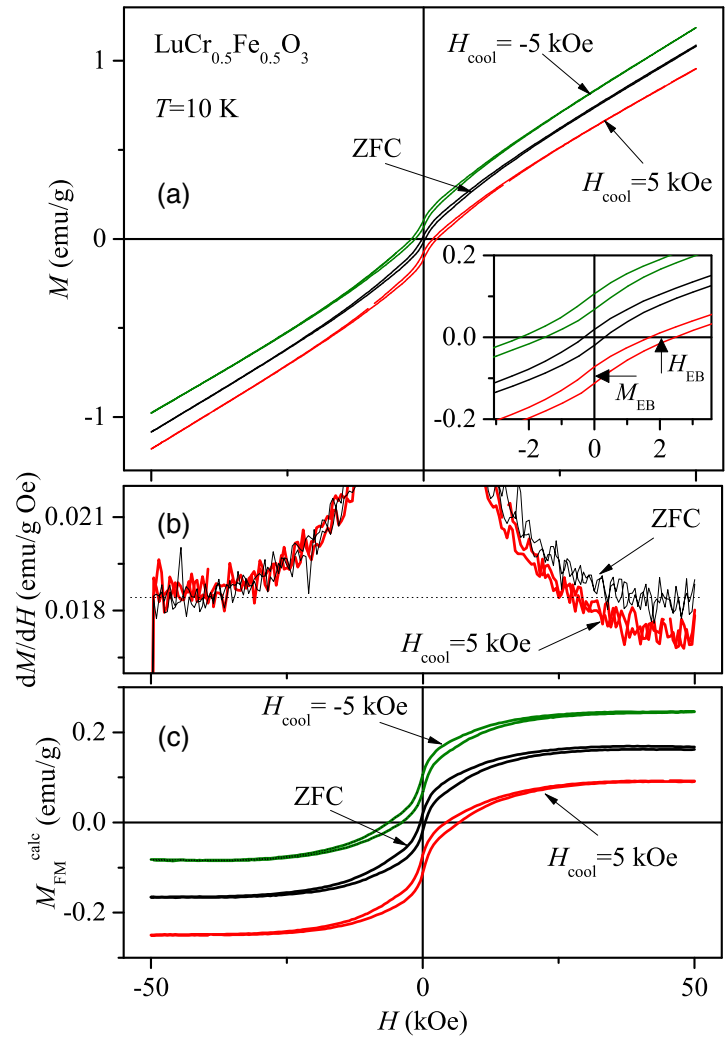

FIG. 4. (a) Magnetization hysteresis loops of $\mathrm{LuFe}_{0.5} \mathrm{Cr}_{0.5} \mathrm{O}_{3}$ at $10 \mathrm{~K}$ measured between $\pm 50 \mathrm{kOe}$ for $\mathrm{ZFC}$ mode and for cooling fields $H_{\text {cool }}=+5 \mathrm{kOe}$ and $-5 \mathrm{kOe}$. Inset shows the loops in an extended scale. (b) High-field derivatives $d M / d H=\chi_{\mathrm{HF}}$ vs $H$ for the cases $H_{\text {cool }}=0$ and $5 \mathrm{kOe}$. (c) FM components $M_{\mathrm{FM}}^{\text {calc }}$ of the loops presented in panel (a) calculated by subtracting the AFM component according to the equation $M_{\mathrm{FM}}^{\text {calc }}(H)=M(H)-\chi_{\mathrm{HF}} H$.

which can be reversed by varying $H_{\text {cool }}$ [40], whereas the positive EB is a prerequisite for spontaneous magnetization reversal [41]. Recently, the positive EB corresponding to the negative magnetization around $T_{\text {comp }}$ was observed in the $\mathrm{ErFeO}_{3}$ single crystal [9].

Let us discuss a possible origin of the EB anisotropy and unusual highly nontrivial behavior of both $\mathrm{ZFC}$ and FC magnetization curves of $\mathrm{LuFe}_{0.5} \mathrm{Cr}_{0.5} \mathrm{O}_{3}$. The magnetic system of the studied sample, frozen in its demagnetized state following the ZFC mode, represents an ensemble of weak FM moments pointed in random directions. Hence, the ZFC magnetization is positive (see Fig. 2) exhibiting mainly the contribution from the AFM component while the FM moments are compensated, and the EB anisotropy is averaged to zero, leading to the usual symmetric ZFC loop with positive remanence [inset in Fig. 4(a)]. In contrast, when the sample is cooled through $T_{\mathrm{N}}$ in magnetic field $H_{\text {cool }}$, the weak FM moments resulting from the canted AFM structure tend to align in the field direction. Note that such FC induced moment alignment is possible even at moderate $H_{\text {cool }}$, owing to the small anisotropy fields at temperatures around $T_{\mathrm{N}}$. Consequently, the magnetic system frozen at $10 \mathrm{~K}$ exhibits the oriented FM moments dominated by the $\mathrm{Cr}$ spins, therefore, aligned oppositely to the applied field. This is a source of the negative FC magnetization (Fig. 2) and 
positive EB anisotropy evidenced by a shift of the FC loop towards positive fields.

The field dependence of derivatives $d M\left(H, H_{\text {cool }}\right) / d H=$ $\chi_{\mathrm{HF}}$ at high applied fields, up to $50 \mathrm{kOe}$ for the cases $H_{\text {cool }}=0$ (ZFC mode) and $+5 \mathrm{kOe}$, is shown in Fig. 4(b). Both the $\mathrm{ZFC}$ and $\mathrm{FC}$ curves reveal saturation in fields near $50 \mathrm{kOe}$. However, in the case of the FC mode with $H_{\text {cool }}=+5 \mathrm{kOe}$ the magnetic susceptibility $\chi_{\mathrm{HF}}$, measured at negative field, that is in the direction of $M_{\mathrm{r}}$, coincides with that measured in the ZFC mode, while $\chi_{\mathrm{HF}}$, measured in the case of the same FC mode at positive applied field, that is in the direction opposite to $M_{\mathrm{r}}$, is by $\sim 6 \%$ smaller at $H=50 \mathrm{kOe}$ than that measured at positive applied field in the ZFC mode (on the contrary, in the case of $H_{\text {cool }}=-5 \mathrm{kOe}$, the $\chi_{\mathrm{HF}}$ appears to be larger at positive fields). Naturally, the $\chi_{\mathrm{HF}} \mathrm{S}$ are identical for both opposite field directions in ZFC mode. The puzzling divergence of the $\chi_{\mathrm{HF}} \mathrm{S}$ measured at positive/negative applied field in the FC mode testifies that the applied field being as strong as $50 \mathrm{kOe}$ is insufficient to order the weak FMs. In other words, the field of $50 \mathrm{kOe}$ is smaller than the maximal FM overturning field in the studied polycrystalline sample.

The field-dependent weak FM contribution to magnetization $M_{\mathrm{FM}}(H)$ may be derived from the magnetization loops, based on the approximation that magnetization $M\left(H, H_{\text {cool }}\right)$ is composed of two contributions, a linear field-dependent AFM contribution, $\chi_{\mathrm{HF}} H$, where $\chi_{\mathrm{HF}}$ is the $M\left(H, H_{\mathrm{cool}}\right)$ slope at high fields, and a field-dependent FM contribution from canted AFM structure $M_{\mathrm{FM}}\left(H, H_{\text {cool }}\right)$ as follows: $M\left(H, H_{\text {cool }}\right)=$ $\chi_{\mathrm{HF}} H+M_{\mathrm{FM}}\left(H, H_{\text {cool }}\right)$. Strictly speaking, this relation should be valid for applied field higher than the maximal FM overturning field in a polycrystalline sample. A very similar procedure applied to separate a tiny FM cluster phase response, covered by the background signal of the dominant AFM matrix, has been performed for the phase-separated charge-ordered $\mathrm{Pr}_{1 / 3} \mathrm{Ca}_{2 / 3} \mathrm{MnO}_{3}$ EB system, exhibiting negative exchangebias field $H_{\mathrm{EB}}$ and positive remanence asymmetry $M_{\mathrm{EB}}$ [37]. Figure $4(\mathrm{c})$ presents the $\mathrm{FM} M_{\mathrm{FM}}^{\text {calc }}\left(H, H_{\text {cool }}\right)$ curves determined by subtracting the AFM component with $\chi_{\mathrm{HF}}$, calculated at $H=50 \mathrm{kOe}$, from the original loops, shown in Fig. 4(a), according to the equation $M_{\mathrm{FM}}^{\text {calc }}\left(H, H_{\text {cool }}\right)=M\left(H, H_{\text {cool }}\right)-$ $\chi_{\mathrm{HF}} H$. As expected, the $M_{\mathrm{FM}}^{\text {calc }}\left(H, H_{\text {cool }}\right)$ curves for the FC mode with $H_{\text {cool }}=5$ and $-5 \mathrm{kOe}$ can be sufficiently well reproduced by a shift of the $M_{\mathrm{FM}}^{\text {calc }}(H, 0)$ curve for the ZFC mode by the values of -0.08 and $+0.08 \mathrm{emu} / \mathrm{g}$, respectively, that means by the values close to $M_{\mathrm{r}}(10 \mathrm{~K})$ [Fig. 3(a)]. Both the ZFC and FC loops reveal visual saturation in fields $\sim 30 \mathrm{kOe}$ that would support the assumption regarding the high-field regime. However, the specific symmetric shift of the FC loops with regard to ZFC loops together with the peculiar behavior of the susceptibilities near $50 \mathrm{kOe}$ demonstrates that actually spin-flip fields are not reached and the magnetization curves shown in Fig. 4 most likely reflect a contribution of the fieldinduced spin-reorientation transitions mainly in crystallites with the FIM moment oriented parallel to the field direction. The spin-flip transition in crystallites, in which after cooling the low-temperature FIM moment is oriented oppositely to the applied field, takes place at strong fields, probably much larger than $50 \mathrm{kOe}$, due to small magnitude of the FIM moment. Namely, such crystallites are characterized by lower susceptibility due to opposite orientations of the applied field and the Dzyaloshinskii field.

In order to understand better the FC induced polarization of the weak FM moments in $\mathrm{LuFe}_{0.5} \mathrm{Cr}_{0.5} \mathrm{O}_{3}$, the influence of the cooling field up to $H_{\text {cool }}=90 \mathrm{kOe}$ on the EB parameters $H_{\mathrm{EB}}$ and $M_{\mathrm{EB}}$ was studied at 10 and $150 \mathrm{~K}$. Figure 5(a) shows the central part of the $M(H)$ hysteresis loops at $10 \mathrm{~K}$ recorded with various $H_{\text {cool }}$. It appears that the $H_{\mathrm{EB}}$ and $\left|M_{\mathrm{EB}}\right|$ first sharply increase with increasing $H_{\text {cool }}$, and maximize at relatively small field $\sim 1 \mathrm{kOe}$, both at 10 and $150 \mathrm{~K}$ [see Figs. 5(c) and 5(d)]. This behavior verifies that during the FC, the weak FM moments' reorientation to the field direction happens at temperatures around $T_{\mathrm{N}}$, at which the anisotropy fields are small and therefore even a small magnetic field is sufficient to overcome an anisotropy energy barrier. Hence, the alignment degree of the FM moments increases with increasing $H_{\text {cool }}$, and for the moderate $H_{\text {cool }}$ values between 1 and $15 \mathrm{kOe}$ the hysteresis loops $M(H)$ show the maximal field $H_{\mathrm{EB}}$ and remanence asymmetry $M_{\mathrm{EB}}$, that may be related to a maximal ordering of the weak FM moments. Intriguingly, with further increasing $H_{\text {cool }}$, both loop shifts, $H_{\mathrm{EB}}$ and $M_{\mathrm{EB}}$, gradually decrease, and at $H_{\text {cool }}=90 \mathrm{kOe}$ the loop appears to locate near the origin [Fig. 4(a)]. The FM components $M_{\mathrm{FM}}^{\text {calc }}$ of the loops with $H_{\text {cool }}=+50$ and $+90 \mathrm{kOe}$ [Fig. 5(b)], calculated in manner described above, look like those obtained for much smaller cooling fields $H_{\text {cool }}=+5 \mathrm{kOe}$ [Fig. 4(c)]. However, they are shifted with regard to the ZFC loop by smaller values $M_{\mathrm{FM}}^{\text {calc }}\left(0, H_{\text {cool }}\right) \approx-0.06$ and $-0.02 \mathrm{emu} / \mathrm{g}$, respectively, that is close to respective remnant FM moments.

Let us compare the cooling field dependencies of $H_{\mathrm{EB}}$ and $M_{\text {EB }}$ presented in Figs. 5(c) and 5(d) with those obtained recently for isostructural $\mathrm{YFe}_{0.5} \mathrm{Cr}_{0.5} \mathrm{O}_{3}$ [27-29]. Unfortunately, a clear comparison is not conceivable, because of very different $H_{\mathrm{EB}}$ vs $H_{\text {cool }}$ data of Refs. [27,28], obtained using a low value of field $H_{\max }=5 \mathrm{kOe}$. Nevertheless, the $H_{\mathrm{EB}}$ vs $H_{\text {cool }}$ dependencies were found to be very similar in both $\mathrm{Lu}$ and Y compounds when measured with $H_{\text {cool }}$ smaller than $5 \mathrm{kOe}$ (see Ref. [28]) while they appear strongly different at higher $H_{\text {cool }}$ [29]. Moreover, the $H_{\mathrm{EB}}$ in $\mathrm{YFe}_{0.5} \mathrm{Cr}_{0.5} \mathrm{O}_{3}$ measured at different maximal field $H_{\max }$ of $5 \mathrm{kOe}$ [28] and $30 \mathrm{kOe}$ [29] shows comparable values, implying a small minor loop effect. Because of the importance of the minor loop impact, discussed in the Introduction, we have checked this issue for $\mathrm{LuFe}_{0.5} \mathrm{Cr}_{0.5} \mathrm{O}_{3}$, measuring the $H_{\mathrm{EB}}$ and $M_{\mathrm{EB}}$ vs $H_{\text {max }}$ dependencies at $150 \mathrm{~K}$ with $H_{\text {cool }}=5 \mathrm{kOe}$; see inset in Fig. 5(d). It appears that EB diminishes almost linearly, by $\sim 12 \%$ only, with increasing $H_{\max }$ from 10 to $90 \mathrm{kOe}$. In addition, a minor reduction in EB was found also at higher $H_{\text {cool }}$; see black solid symbols in Figs. 5(c) and 5(d). These results suggest that the minor loop effect is not crucial in $\mathrm{Lu}$ and $\mathrm{Y}$ ferrite-chromite FIMs, in strong contrast to that in conventional EB FM-AFM phase-separated systems [33-35]. On the other hand, for both compounds, the $H_{\mathrm{EB}}$ vs $H_{\text {cool }}$ dependence obtained at small $H_{\text {cool }}$ looks like that observed for FM-AFM phase-separated systems [42]. In view of that, we have compared the $H_{\mathrm{EB}}\left(H_{\text {cool }}\right)$ dependence for $\mathrm{LuFe}_{0.5} \mathrm{Cr}_{0.5} \mathrm{O}_{3}$, obtained for restricted region $0<H_{\text {cool }}<5 \mathrm{kOe}$, with the theoretical one, proposed in Ref. [37] for phase-separated systems consisting of single-domain 

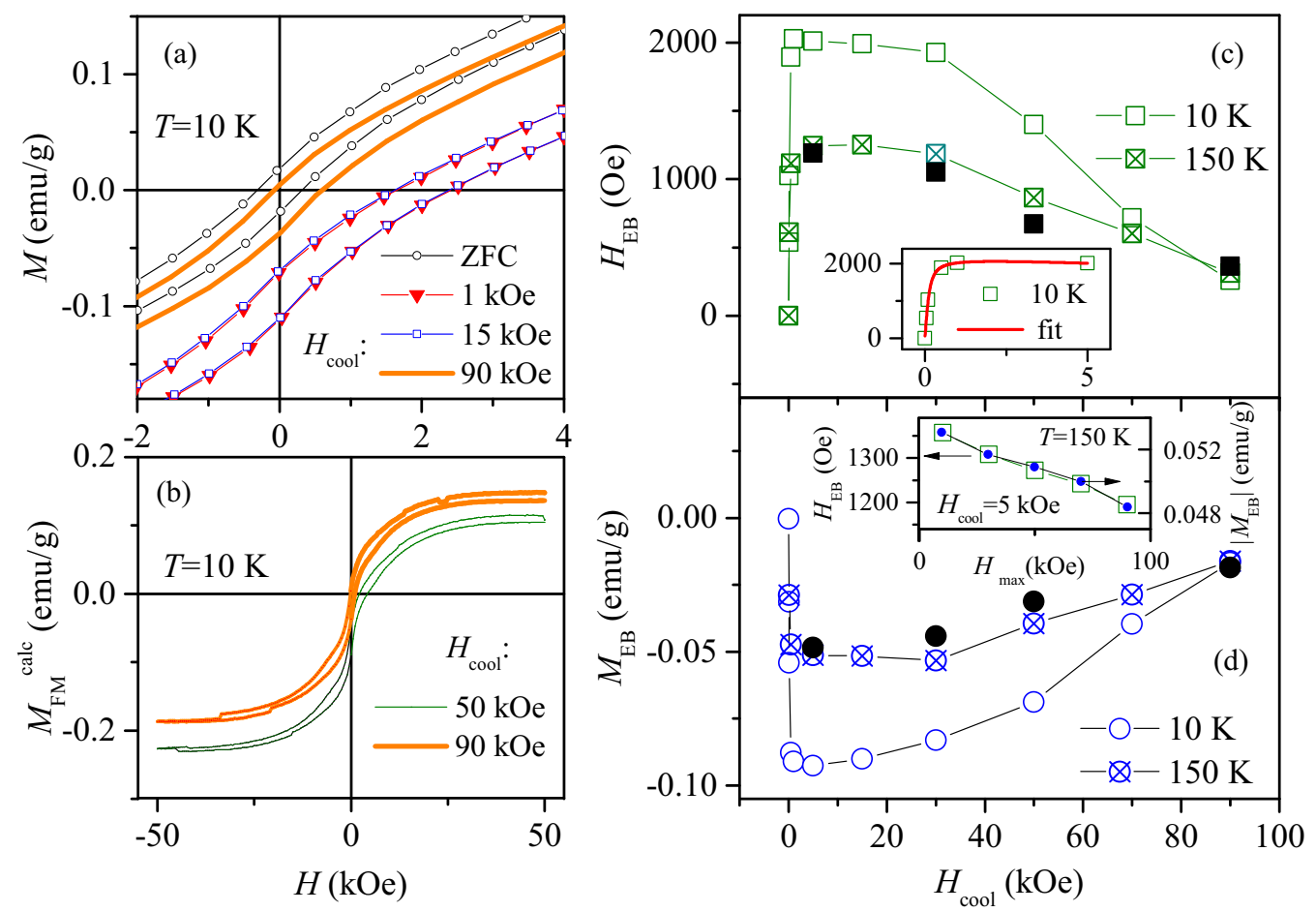

FIG. 5. (a) Magnetization hysteresis loops of $\mathrm{LuFe}_{0.5} \mathrm{Cr}_{0.5} \mathrm{O}_{3}$ shown in extended scale, measured between $\pm 50 \mathrm{kOe}$ at $10 \mathrm{~K}$ for different cooling fields $H_{\text {cool }}$. (b) FM components $M_{\mathrm{FM}}^{\text {calc }}$ of the loops with $H_{\text {cool }}=50 \mathrm{kOe}$ and $90 \mathrm{kOe}$, calculated by subtracting the AFM component according to the equation $M_{\mathrm{FM}}^{\text {calc }}=M-\chi_{\mathrm{HF}} H$. Cooling field dependencies of EB field $H_{\mathrm{EB}}$ (c) and remanence asymmetry $M_{\mathrm{EB}}(\mathrm{d})$ at $10 \mathrm{~K}$ and $150 \mathrm{~K}$. Solid symbols show the EB parameters derived from the loops measured between $\pm 90 \mathrm{kOe}$ at $150 \mathrm{~K}$. Inset of (c) presents successful fit of Eq. (1) to the $H_{\mathrm{EB}}$ vs $H_{\text {cool }}$ dependence for low cooling fields. Inset of (d) shows the linear decrease of both $H_{\mathrm{EB}}$ and $\left|M_{\mathrm{EB}}\right|$ with increasing maximal measuring field $H_{\max }$ at $150 \mathrm{~K}$.

FM clusters embedded in the AFM matrix:

$$
H_{\mathrm{EB}} \propto J\left\{\left[J \mu_{0} /\left(g \mu_{\mathrm{B}}\right)^{2}\right] L\left(\mu H_{\text {cool }} / k_{\mathrm{B}} T_{\mathrm{f}}\right)+H_{\text {cool }}\right\},
$$

where $J$ is the interface exchange constant, $g=2$ is the gyromagnetic factor, $\mu_{\mathrm{B}}$ is the Bohr magneton, $L$ denotes the Langevin function, $\mu_{0}=4 \mu_{\mathrm{B}}$ is the average magnetic moment of $\mathrm{Fe}$ and $\mathrm{Cr}$ spins per molecule, $\mu=N \mu_{0}$ is the magnetic moment of the FM clusters with $N$ number of spins, and $T_{\mathrm{f}}=270 \mathrm{~K}$ is the temperature below which the EB appears. Equation (1) has been frequently used for evaluation of the FM cluster size in a variety of EB phase-separated systems, such as manganites and cobaltites [42]. The solid line in the inset of Fig. 5(c) represents the best fit with Eq. (1) obtained for the values of fitting parameters $N=(2.1 \pm 0.06) \times 10^{6}$ and $J / k_{\mathrm{B}}=-(4.9 \pm 1) \mathrm{K}$. The calculated value of $N$ corresponds to the FM cluster size of $60 \mathrm{~nm}$, and the interfacial exchange interaction $J$ is of the AFM nature. Despite the successful fit within small $H_{\text {cool }}$, an attempt of fitting the $H_{\mathrm{EB}}\left(H_{\text {cool }}\right)$ dependence including fields higher than $5 \mathrm{kOe}$ failed (the errors overcame the fitting parameter values), indicating that the above model [37] linked to the FM-AFM phase-separated systems is inappropriate for our single-phase FIM.

Although the EB is similar in both $\mathrm{Lu}$ and $\mathrm{Y}$ compounds at small $H_{\text {cool }}$, it dramatically differs at high $H_{\text {cool }}$. Namely, EB in $\mathrm{YFe}_{0.5} \mathrm{Cr}_{0.5} \mathrm{O}_{3}$ was found to change sign from positive to negative at $H_{\text {cool }}>10 \mathrm{kOe}$ [29] whereas in $\mathrm{LuFe}_{0.5} \mathrm{Cr}_{0.5} \mathrm{O}_{3}$ it remains positive up to $H_{\text {cool }}=90 \mathrm{kOe}$; see Figs. 5(c) and $5(\mathrm{~d})$. The origin of this discrepancy is puzzling because both exchange and DM interactions in both compounds were estimated to be comparable [20]. Note that the cooling-fieldinduced transition from positive EB to the negative one is opposite to that observed in AFM-FM $\mathrm{FeF}_{2}-\mathrm{Fe}$ bilayers when large enough $H_{\text {cool }}$ overcomes the AFM interfacial coupling [38,39]. Nevertheless, such transition at $H_{\text {cool }} \sim 100$ kOe has been observed recently in ferrimagnetic core/shell nanoparticles with AFM interfacial coupling, based on Fe and Mn oxides [43].

It should be noted that the above scenario of the FC induced EB anisotropy in the $\mathrm{LuFe}_{0.5} \mathrm{Cr}_{0.5} \mathrm{O}_{3}$ polycrystalline sample resembles the mechanisms of the emerging $\mathrm{EB}$ in the usual FM/AFM interface exchange-biased systems. In the studied FIM, the cooling field $H_{\text {cool }}$ leads to the field-induced alignment of the weak FM moments, i.e., the formation of a clear FIM structure with the FM easy axis along the applied field, and in the case of the usual FM/AFM interface the $H_{\text {cool }}$ induces a similar FM alignment of uncompensated AFM spins at the interface, giving rise to the unidirectional anisotropy. Therefore, in $\mathrm{LuFe}_{0.5} \mathrm{Cr}_{0.5} \mathrm{O}_{3}$ single crystals with the FIM structure and with the FM easy axis along the field $H$, one can expect that the EB is not dependent on the $H_{\text {cool }}$ value (of course, in the range of moderate applied fields), as has been observed in $\mathrm{ErFeO}_{3}$ single crystal [9].

Additionally, we have examined the magnetic training effect (TE) for $\mathrm{EB}$ in $\mathrm{LuFe}_{0.5} \mathrm{Cr}_{0.5} \mathrm{O}_{3}$, which is considered an important tool to bring information about the nature of EB. In traditional exchange-biased FM-AFM interface systems $[2,3,37]$, the TE refers to the gradual decrease of the EB field $H_{\mathrm{EB}}$ under an increasing number of consecutively 

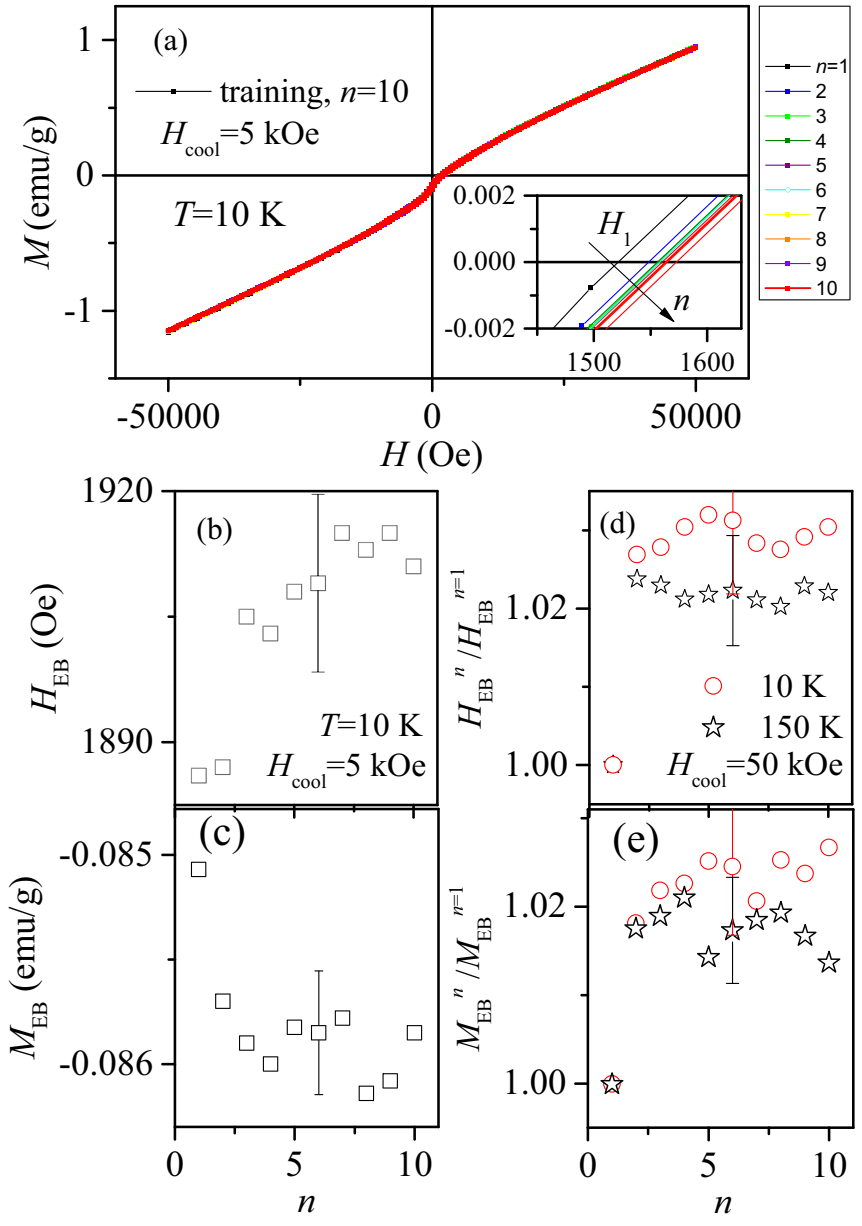

FIG. 6. (a) The $M(H)$ loops performed consecutively ten times between $\pm 50 \mathrm{kOe}$ at $10 \mathrm{~K}$ after cooling in field of $5 \mathrm{kOe}$. The inset shows variation of the first coercive field $H_{1}$ with increasing loop number $n$. (b), (c) The field $H_{\mathrm{EB}}$ and remanence asymmetry $M_{\mathrm{EB}}$ at $10 \mathrm{~K}$, obtained with $H_{\text {cool }}=5 \mathrm{kOe}$, versus increasing number of recurrent hysteresis loops. (d), (e) Relative change in $H_{\mathrm{EB}}$ and $M_{\mathrm{EB}}$ during magnetic training between $\pm 50 \mathrm{kOe}$ with $H_{\text {cool }}=50 \mathrm{kOe}$ at $10 \mathrm{~K}$ and $150 \mathrm{~K}$

performed hysteresis loops at a fixed temperature, and the origin of TE is generally linked to the spin rearrangement at the interface [44,45]. Figure 6(a) presents the $M(H)$ loops performed consecutively ten times between $\pm 50 \mathrm{kOe}$ at 10 $\mathrm{K}$ after $\mathrm{FC}$ with $H_{\text {cool }}=5 \mathrm{kOe}$. They show that EB does not diminish as usually observed in traditional FM/AFM systems, but on the contrary, both $H_{\mathrm{EB}}$ and $\left|M_{\mathrm{EB}}\right|$ increase slightly during the training [see Figs. 6(b) and 6(c)]. Very similar behavior was found also for training with $H_{\text {cool }}=50 \mathrm{kOe}$ and $H_{\max }=50 \mathrm{kOe}$ performed at temperatures 10 and $150 \mathrm{~K}$ [see Figs. 6(d) and 6(e)]. It appears that the apparent changes in EB occur between the first and the second loops, due to the large shift of the descending branch [see inset in Fig. 6(a)], while the EB changes induced by the next hysteresis cycles are within experimental errors. Such type of TE, representing absence of the thermally activated magnetization processes, is identified as athermal training [44]. The athermal TE leading to the increase of the positive EB has been first observed in $\mathrm{GdFe} / \mathrm{TbFe}$ ferrimagnetic bilayers exhibiting a transition from
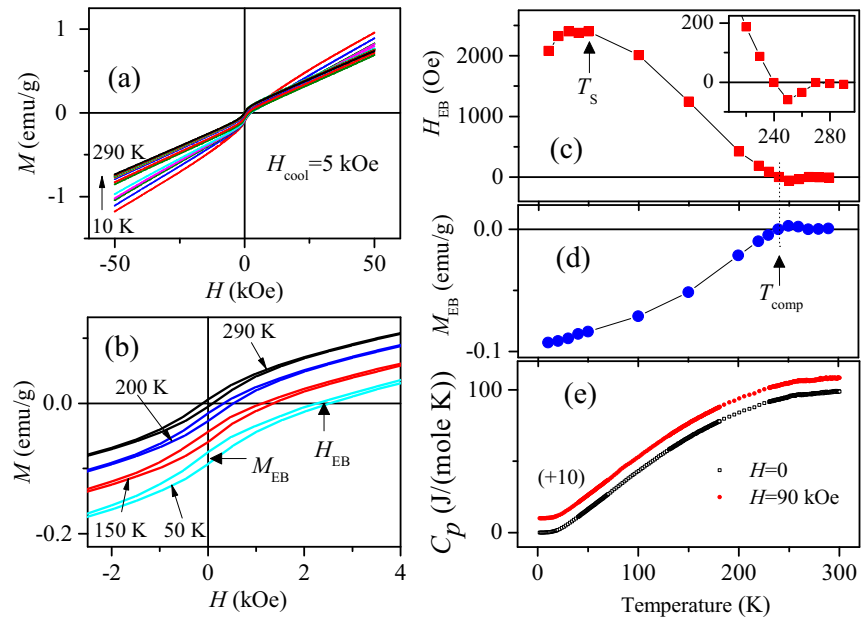

FIG. 7. (a) Magnetization hysteresis loops of $\mathrm{LuFe}_{0.5} \mathrm{Cr}_{0.5} \mathrm{O}_{3}$ measured between $\pm 50 \mathrm{kOe}$ with cooling field $H_{\text {cool }}=5 \mathrm{kOe}$ at different temperatures. (b) Some loops presented in (a) shown in an extended scale. Temperature dependencies of EB field $H_{\mathrm{EB}}$ (c) and remanence asymmetry $M_{\mathrm{EB}}$ (d). Inset in (c), presenting the enlargement of the curve of the main panel in the vicinity of $T_{\text {comp }}$, shows that EB is positive below $T_{\text {comp }}$ and negative above $T_{\text {comp }}$. (e) Temperature dependencies of specific heat of $\mathrm{LuFe}_{0.5} \mathrm{Cr}_{0.5} \mathrm{O}_{3}$ measured in zero magnetic field and in the field of $90 \mathrm{kOe}$. The curve measured in magnetic field is shifted along the $C_{\mathrm{p}}$ axis by the value given in parentheses.

negative to positive EB with increasing $H_{\text {cool }}$ [46]. It was suggested that the GdFe magnetization reversal, at the first cycle, induces a new spin configuration in the TbFe layer, to minimize the energy, and it remains unchanged with additional field cycling [46]. The increase of the positive EB during TE was further observed in antiferromagnetic $\mathrm{NiFe} / \mathrm{IrMn}$ bilayers [44]. In contrast, no TE was noticed in the case of negative EB in $\mathrm{GdFe} / \mathrm{TbFe}$ bilayers [46]. Similarly, we have not found any visible TE for the case of negative $\mathrm{EB}$ (data not shown), which occurs in $\mathrm{LuFe}_{0.5} \mathrm{Cr}_{0.5} \mathrm{O}_{3}$ at temperatures between $T_{\text {comp }}$ and $T_{\mathrm{N}}$. According to the TE results, the positive EB anisotropy, once induced under FC and frozen at low temperature, is very stable and remains unchanged after numerous field cycles within $\pm 50 \mathrm{kOe}$. Both the FM moment, directed against the applied field, and $H_{\mathrm{EB}}$ even increase slightly, by $\sim 2 \%$, after the first magnetization cycle. Lack of the typical TE suggests an unusual $\mathrm{EB}$ nature in the $\mathrm{LuFe}_{0.5} \mathrm{Cr}_{0.5} \mathrm{O}_{3}$ ferrimagnet.

Magnetization hysteresis loops of $\mathrm{LuFe}_{0.5} \mathrm{Cr}_{0.5} \mathrm{O}_{3}$ measured between $\pm 50 \mathrm{kOe}$ with cooling field $H_{\text {cool }}=5 \mathrm{kOe}$ at different temperatures between 290 and $10 \mathrm{~K}$ are presented in Fig. 7(a), while Fig. 7(b) shows the FC loops in an extended scale. The hysteresis in the $M(H)$ loop exists even at $290 \mathrm{~K}$ indicating that the short-range-ordered regions remain well above $T_{\mathrm{N}}$, in accordance with NPD results [26]. Note also that the width of loops, which represents the average coercive field $H_{\mathrm{C}}$, defined as $H_{\mathrm{C}}=\left(H_{2}-H_{1}\right) / 2$, does not change practically in a wide temperature range. The temperature dependencies of EB parameters $H_{\mathrm{EB}}$ and $M_{\mathrm{EB}}$, derived from the $M(H)$ loops, are shown in Figs. 7(c) and 7(d), respectively. One can see that EB is negative above $T_{\text {comp }}=240 \mathrm{~K}$, and it exists even slightly above $T_{\mathrm{N}}$ in agreement with the presence of a 
short-range order, while below $T_{\text {comp }}$ down to $10 \mathrm{~K}$ the EB is positive, representing the field $H_{\mathrm{EB}}>0$ and remanence asymmetry $M_{\mathrm{EB}}<0$. Very similar gradual EB change with reversal of sign across the $T_{\text {comp }}$ has been reported recently for $\mathrm{YFe}_{0.5} \mathrm{Cr}_{0.5} \mathrm{O}_{3}$ [27] and $\mathrm{Y}_{1-x} \mathrm{Ho}_{x} \mathrm{Fe}_{0.5} \mathrm{Cr}_{0.5} \mathrm{O}_{3}(x=0,0.05$, 0.1 ) [29] ferrite-chromites, and also for other FIM systems presenting a magnetization reversal, such as $\mathrm{Sr}_{2} \mathrm{YbRuO}_{6}$ double perovskite [6] and $\mathrm{La}_{0.2} \mathrm{Ce}_{0.8} \mathrm{CrO}_{3}$ nanoparticles containing a FM Co core and AFM CoO shell [47]. In contrast, several exchange-biased FIMs, such as GdCo-Co multilayers [14], and $\mathrm{Nd}_{0.75} \mathrm{Ho}_{0.25} \mathrm{Al}_{2}$ [4], $\mathrm{Co}\left(\mathrm{Cr}_{0.95} \mathrm{Fe}_{0.05}\right) \mathrm{O}_{4}$ [8], $\mathrm{ErFeO}_{3}$ [9], and $\mathrm{TmCrO}_{3}$ [48] single-phase materials show very different behavior at $T_{\text {comp }}$; namely, EB practically disappears far from $T_{\text {comp }}$, but it increases and diverges approaching $T_{\text {comp }}$, and

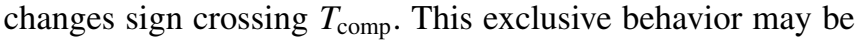
understood within the model proposed by Webb et al. [14] for simple FIM comprising two AFM coupled sublattices, A and B, with opposite magnetic moments. According to this model, the EB field is inversely proportional to the net magnetic moment $\left(M_{\mathrm{A}}-M_{\mathrm{B}}\right)$; therefore, $H_{\mathrm{EB}}$ becomes noticeable around $T_{\text {comp }}$ only, when moment $\left(M_{\mathrm{A}}-M_{\mathrm{B}}\right)$ becomes small enough, and it diverges at $T_{\text {comp }}$. Moreover, the sign of $H_{\mathrm{EB}}$ is determined by the direction of the moment $\left(M_{\mathrm{A}}-M_{\mathrm{B}}\right)$ with respect to the applied field. Since $\mathrm{LuFe}_{0.5} \mathrm{Cr}_{0.5} \mathrm{O}_{3}$ shows very different temperature dependence of $\mathrm{EB}$, we assume that it has a more complicated magnetic structure than that considered in the simple Webb model, probably due to the presence of the competing DM interactions in ferrite-chromites.

In order to verify the interpretations presented above, supplementary specific heat studies were performed. Specific heat of the $\mathrm{LuFe}_{0.5} \mathrm{Cr}_{0.5} \mathrm{O}_{3}$ pellet, used previously in the magnetization studies, was measured from $2 \mathrm{~K}$ to $300 \mathrm{~K}$ in zero magnetic field and in the field of $90 \mathrm{kOe}$. The measured dependencies presented in Fig. 7(e) show that the anomaly related to the magnetic phase transition at $\sim 260 \mathrm{~K}$ is very weak and smeared, which is consistent with the results of the magnetization studies that revealed the presence of the short-range magnetic ordering up to $290 \mathrm{~K}$. No anomaly was observed in the zero-field temperature dependence of specific heat at $T_{\text {comp }}$. This is consistent with theoretical predictions that in zero magnetic field the compensation point [49] is related neither to any change of the system symmetry nor to any phase transition. On the other hand, in nonzero magnetic field, spin reorientation transitions are expected to appear near $T_{\text {comp }}$ at relatively low field. Thus, it is rather strange that no anomalies related to such transitions were observed in the dependence measured in the field of $90 \mathrm{kOe}$. This can be attributed to the fact that in the polycrystalline sample, crystallographic axes of particular grains are oriented randomly with respect to the direction of the applied field and thus such spin reorientation transition appears at different temperatures in particular grains, which makes these small specific heat anomalies unnoticeable in the temperature dependence of the specific heat of the whole sample. The same explanation can be assumed for the lack of a specific heat anomaly at $T_{\mathrm{S}}$.

\section{CONCLUSIONS}

In summary, the exchange-bias effect was found and investigated in the weak ferrimagnet $\mathrm{LuFe}_{0.5} \mathrm{Cr}_{0.5} \mathrm{O}_{3}$ with two canted Fe and $\mathrm{Cr}$ AFM sublattices with oppositely directed weak FM moments, signified at low temperatures by the magnetic moment reversal and negative magnetization. It was found that the field-cooled magnetization hysteresis loops show the EB effect, namely both vertical and horizontal shifts from the origin. The EB is positive below $T_{\text {comp }}$ and negative above $T_{\text {comp }}$ due to weak ferrimagnetic behavior of $\mathrm{LuFe}_{0.5} \mathrm{Cr}_{0.5} \mathrm{O}_{3}$ permitting the magnetic moment reversal governed by a specific competition of the DM interactions between $\mathrm{Fe}^{3+}$ and $\mathrm{Cr}^{3+}$ ions.

Specific symmetric shift of the FC loops with regard to ZFC loops together with peculiar behavior of the susceptibilities near $50 \mathrm{kOe}$ demonstrate that actually the maximal FM overturning fields are not reached and magnetization curves most likely reflect a contribution of the field-induced spinreorientation transitions near $T_{\mathrm{S}} \approx 50 \mathrm{~K}$, mainly in crystallites with the weak FM moment oriented parallel to the field direction. Full FM overturning in crystallites with FIM moment oriented oppositely to applied field takes place at very strong field, probably much larger than $50 \mathrm{kOe}$, due to small magnitude of the FIM moment. Such crystallites are characterized by a lower susceptibility due to opposite orientations of the applied field and the Dzyaloshinskii field. The training procedure shows that the EB field does not decrease with increasing number of cycles as usually observed in traditional FM/AFM systems. On the contrary, it increases slightly, pointing to the stability of the EB observed. It should be noted also that the variety of such extraordinary properties as high compensation temperature $T_{\text {comp }}=240 \mathrm{~K}$, temperature-controlled positive/negative EB below/above $T_{\text {comp }}$, and switching of the magnetization direction to the opposite one by magnetic field without changing its polarity makes the $\mathrm{LuFe}_{0.5} \mathrm{Cr}_{0.5} \mathrm{O}_{3}$ ferrimagnet a promising candidate for application in magnetic memories.

\section{ACKNOWLEDGMENTS}

This work was partially supported by Polish NCN Grant No. 2014/15/B/ST3/03898 and by the European Union, within the European Regional Development Fund, through Innovative Economy Grant No. POIG.01.01.02-00-108/09. A.S.M. thanks a support by Act 211 Government of the Russian Federation, Contract No. 02.A03.21.0006 and by the Ministry of Education and Science of the Russian Federation, Projects No. 2277 and No. 5719.
[1] W. H. Meiklejohn and C. P. Bean, Phys. Rev. 102, 1413 (1956); 105, 904 (1957).

[2] J. Nogués, J. Sort, V. Langlais, V. Skumryev, S. Suriñach, J. S. Muñoz, and M. D. Baró, Phys. Rep. 422, 65 (2005).
[3] F. Radu and H. Zabel, in Magnetic Heterostructures: Advances and Perspectives in Spinstructures and Spintransport, edited by H. Zabel and S. D. Bader, Springer Tracts in Modern Physics, Vol. 227 (Springer-Verlag, Berlin, 2008), pp. 97-184. 
[4] P. D. Kulkarni, A. Thamizhavel, V. C. Rakhecha, A. K. Nigam, P. L. Paulose, S. Ramakrishnan, and A. K. Grover, Europhys. Lett. 86, 47003 (2009).

[5] S. Venkatesh, U. Vaidya, V. C. Rakhecha, S. Ramakrishnan, and A. K. Grover, J. Phys.: Condens. Matter 22, 496002 (2010).

[6] R. P. Singh, C. V. Tomy, and A. K. Grover, Appl. Phys. Lett. 97, 182505 (2010).

[7] K. Yoshii, Appl. Phys. Lett. 99, 142501 (2011).

[8] R. Padam, S. Pandya, S. Ravi, A. K. Nigam, S. Ramakrishnan, A. K. Grover, and D. Pal, Appl. Phys. Lett. 102, 112412 (2013).

[9] I. Fita, A. Wisniewski, R. Puzniak, V. Markovich, and G. Gorodetsky, Phys. Rev. B 93, 184432 (2016).

[10] A. K. Nayak, M. Nicklas, S. Chadov, P. Khuntia, C. Shekhar, A. Kalache, M. Baenitz, Yu. Skourski, V. K. Guduru, A. Puri, U. Zeitler, J. M. D. Coey, and C. Felser, Nat. Mater. 14, 679 (2015).

[11] P. Nordblad, Nat. Mater. 14, 655 (2015).

[12] A. Wisniewski, I. Fita, R. Puzniak, and V. Markovich, Exchange bias effect in bulk perovskite manganites, in Exchange Bias: From Thin Film to Nanogranular and Bulk Systems, edited by Surender Kumar Sharma (CRC Press, Taylor and Francis Group, 2017).

[13] I. L. Prejbeanu, M. Kerekes, R. C. Sousa, H. Sibuet, O. Redon, B. Dieny, and J. P. Nozičres, J. Phys.: Condens. Matter 19, 165218 (2007).

[14] D. J. Webb, A. F. Marshall, A. M. Toxen, T. H. Geballe, and R. M. White, IEEE Trans. Magn. 24, 2013 (1988).

[15] A. M. Kadomtseva, A. S. Moskvin, I. G. Bostrem, B. M. Wanklyn, and N. A. Khafizova, Zhurnal experimentalnoi i teoreticheskoi fiziki 72, 2286 (1977) [JETP 45, 1202 (1977)].

[16] E. V. Sinitsyn, A. M. Kadomtseva, A. S. Moskvin, I. G. Bostrem, V. A. Timofeeva, and O. V. Kazakevich, Fizika Tverdogo Tela 25, 285 (1983) [Sov. Phys. Solid State 25, 161 (1983)].

[17] A. M. Kadomtseva, A. P. Agafonov, I. A. Zorin, A. S. Moskvin, T. L. Ovchinnikova, and V. A. Timofeeva, Zhurnal experimentalnoi i teoreticheskoi fiziki 84, 1432 (1983) [JETP 57, 833 (1983)].

[18] A. M. Kadomtseva and A. S. Moskvin, Acta Phys. Pol. A 68, 303 (1985).

[19] A. S. Moskvin, N. S. Ovanesyan, and V. A. Trukhtanov, Hyperfine Interact. 1, 265 (1975).

[20] O. V. Billoni, F. Pomiro, S. A. Cannas, C. Martin, A. Maignan, and R. E. Carbonio, J. Phys.: Condens. Matter 28, 476003 (2016).

[21] A. S. Moskvin and I. G. Bostrem, Fizika Tverdogo Tela 19, 2616 (1977) [Sov. Phys. Solid State 19, 1532 (1977)].

[22] A. S. Moskvin, J. Magn. Magn. Mater. 400, 117 (2016).

[23] A. K. Azad, A. Mellergard, S. G. Eriksson, S. A. Ivanov, S. M. Yunus, F. Lindberg, G. Svensson, and R. Mathieu, Mater. Res. Bull. 40, 1633 (2005).

[24] J. Mao, Y. Sui, X. Zhang, Y. Su, X. Wang, Z. Liu, Y. Wang, R. Zhu, Y. Wang, W. Liu, and J. Tang, Appl. Phys. Lett. 98, 192510 (2011).
[25] N. Dasari, P. Mandal, A. Sundaresan, and N. S. Vidhyadhiraja, Europhys. Lett. 99, 17008 (2012).

[26] F. Pomiro, R. D. Sánchez, G. Cuello, A. Maignan, C. Martin, and R. E. Carbonio, Phys. Rev. B 94, 134402 (2016).

[27] J. Mao, Y. Sui, X. Zhang, X. Wang, Y. Su, Z. Liu, Y. Wang, R. Zhu, Y. Wang, W. Liu, and X. Liu, Solid State Commun. 151, 1982 (2011).

[28] M. K. Sharma, K. Singh, and K. Mukherjee, J. Magn. Magn. Mater. 414, 116 (2016).

[29] L. R. Shi, C. X. Wei, Z. Wang, L. Ju, T. S. Xu, T. X. Li, X. W. Yan, and Z. C. Xia, J. Magn. Magn. Mater. 433, 104 (2017).

[30] T. Bora, P. Saravanan, and S. Ravi, J. Supercond. Nov. Magn. 26, 1645 (2013).

[31] T. Bora and S. Ravi, J. Magn. Magn. Mater. 386, 85 (2015).

[32] M. P. Sharannia, S. De, R. Singh, A. Das, R. Nirmala, and P. N. Santhosh, J. Magn. Magn. Mater. 430, 109 (2017).

[33] J. Geshev, J. Appl. Phys. 105, 066108 (2009).

[34] J. Geshev, J. Phys.: Condens. Matter 21, 078001 (2009).

[35] A. Harres, M. Mikhov, V. Skumryev, A. M. H. de Andrade, J. E. Schmidt, and J. Geshev, J. Magn. Magn. Mater. 402, 76 (2016).

[36] E. Lima Jr., T. B. Martins, H. R. Rechenberg, G. F. Goya, C. Cavelius, R. Rapalaviciute, S. Hao, and S. Mathur, J. Magn. Magn. Mater. 320, 622 (2008).

[37] D. Niebieskikwiat and M. B. Salamon, Phys. Rev. B 72, 174422 (2005).

[38] J. Nogues, D. Lederman, T. J. Moran, and I. K. Schuller, Phys. Rev. Lett. 76, 4624 (1996).

[39] J. Nogues, C. Leighton, and I. K. Schuller, Phys. Rev. B 61, 1315 (2000).

[40] I. V. Roshchin, O. Petracic, R. Morales, Z.-P. Li, X. Batlle, and I. K. Schuller, Europhys. Lett. 71, 297 (2005).

[41] Zhi-Pan Li, C. W. Miller, I. V. Roshchin, and I. K. Schuller, Phys. Rev. B 76, 014423 (2007).

[42] S. Giri, M. Patra, and S. Majumdar, J. Phys.: Condens. Matter 23, 073201 (2011).

[43] M. Estrader, A. Lopez-Ortega, S. Estrade, I. V. Golosovsky, G. Salazar-Alvarez, M. Vasilakaki, K. N. Trohidou, M. Varela, D. C. Stanley, M. Sinko, M. J. Pechan, D. J. Keavney, F. Peiro, S. Surinach, M. D. Baro, and J. Nogues, Nat. Commun. 4, 2960 (2013).

[44] A. Hoffmann, Phys. Rev. Lett. 93, 097203 (2004).

[45] S. K. Mishra, F. Radu, H. A. Dürr, and W. Eberhardt, Phys. Rev. Lett. 102, 177208 (2009).

[46] T. Hauet, J. A. Borchers, Ph. Mangin, Y. Henry, and S. Mangin, Phys. Rev. Lett. 96, 067207 (2006).

[47] P. K. Manna, S. M. Yusuf, R. Shukla, and A. K. Tyagi, Appl. Phys. Lett. 96, 242508 (2010).

[48] K. Yoshii, Mater. Res. Bull. 473243 (2012).

[49] A. Szewczyk, A. Dabkowski, S. L. Gnatchenko, N. F. Kharchenko, and B. Krzymanska, Acta Phys. Pol. A 67, 877 (1985). 